Questions de communication

$24 \mid 2013$

Renouvellement des mises en scène télévisuelles de la politique

Frédéric Forest, dir., Les universités en France.

Fonctionnement et enjeux

Mont-Saint-Aignan, Publications des universités de Rouen et du Havre, 2012, 296 pages

Christophe Ippolito

(2) OpenEdition

Journals

Édition électronique

URL : http://journals.openedition.org/questionsdecommunication/8799

DOI : 10.4000/questionsdecommunication.8799

ISSN : 2259-8901

Éditeur

Presses universitaires de Lorraine

Édition imprimée

Date de publication : 31 décembre 2013

Pagination : 282-284

ISBN : 978-2-8143-0182-5

ISSN : $1633-5961$

Référence électronique

Christophe Ippolito, «Frédéric Forest, dir., Les universités en France. Fonctionnement et enjeux »,

Questions de communication [En ligne], 24 | 2013, mis en ligne le 01 février 2014, consulté le 22

septembre 2020. URL : http://journals.openedition.org/questionsdecommunication/8799; DOI :

https://doi.org/10.4000/questionsdecommunication.8799 
exemple, est justement cette difficulté à reconnaitre l'information utile et à bien la faire remonter vers ceux qui en ont réellement besoin.

Puis, ressortentles clivages entre « cousins 》, ces « services intérieurs et extérieurs, civils et militaires » (p. 69) qui se complairaient encore trop dans « leurs domaines habituels (espionnages et terrorisme) » (p. 69) pour se soucier de la criminalité transnationale, alors qu'elle est devenue leur priorité depuis les années 80 au moins. Mais il est vrai que la vision du renseignement, au niveau de «l'ilotier ou agent de quartier, figure centrale d'une police de renseignement » (p. 7I), dont la finalité reste l'arrestation, peut sembler incompatible avec une finalité tout autre, résidant dans l'identification des réseaux et la pénétration des filières pour les démanteler. Et pas nécessairement les juger! Cette incompréhension se trouve contenue dans l'expression « cadre légal »(p. 75), que les auteurs envisagent comme un cadre juridique (pp. 75-77) permettant aux forces de sécurité d'intervenir sur le territoire national alors que la finalité du renseignement extérieur est uniquement le décideur politique ou militaire.

Pourtant écrit par deux spécialistes du genre, de ce plaidoyer en faveur du « renseignement criminel », il ressort un texte mal ciblé. II est trop partiel pour les spécialistes du renseignement, quel que soit leur genre, et trop spécialisé pour un grand public largement ignorant des subtilités du genre. D'autant que, ça et là, apparaissent des allusions au débat public des années Nicolas Sarkozy, comme le « décèlement précoce », un autre concept sorti de la boîte à outil de la « criminologie » signalé comme venant de Xavier Rauffer (p. 53, note 17). En réalité, comme beaucoup de livre du même genre, il reste un livre daté, difficilement utilisable pour le chercheur.

Gérald Arboit

CRULH, université de Lorraine, F-54000 gerald.arboit@aliceadsl.fr

\section{Frédéric FoREST, dir., Les universités en France. Fonctionnement et enjeux.}

Mont-Saint-Aignan, Publications des universités de Rouen et du Havre, 2012,296 p.

L'ouvrage collectif ressemble à son sujet, ce qui ne saurait surprendre dans la mesure où ses auteurs ont tous en commun une expérience administrative significative à l'Université. La moitié des seize administrateurs sont issus du professorat, quatre étant présidents d'université. II aurait pu être intéressant d'intégrer des non-administrateurs, des étudiants (sur leur pratique de l'institution), voire des entrepreneurs ou des consultants (sur leurs interactions avec les universités, le marché de l'emploi, les structures de gouvernance) ou des figures représentatives des grandes écoles. Aussi fallait-il donner la même place (une contribution) à des catégories comme, d'une part, la recherche et les finances et, d'autre part, le patrimoine immobilier, la politique documentaire ou les ressources humaines? On peut en douter. En outre, on reste sur sa faim aussi bien en matière de finances que de recherche. Le lecteur pourra être rebuté par le code administratif qui prévaut dans ce champ, par ce qu'il verra parfois comme de la langue de bois, et par de nombreuses répétitions ; il est invité à préalablement lire la liste des sigles (pp. 285-290) et prendra connaissance avec profit des tableaux qui présentent des données quantitatives fort utiles. Avant tout, l'ouvrage est une synthèse descriptive et ne fait pratiquement aucune place au débat, ou ne s'engage que rarement. II s'agit d'un livre sage ou, comme l'annonce la quatrième de couverture, d'un état des lieux. Mais, si l'on s'attelle à lire sous le code, parfois le simple déplacement d'un mot ou l'ordre des arguments peut signifier un pas de côté, voire une prise de position. Cependant, l'épilogue de l'éditeur oriente la synthèse vers quelques perspectives d'avenir.

L'introduction de Frédéric Forest (pp. 9-10) présente le livre comme un instrument pour des réformes nécessaires. Sous la plume de Christine Musselin, le premier chapitre (pp. 13-25) décrit l'histoire des universités depuis 1789 (mentionnant la suppression des universités sous la Révolution française) jusqu'à aujourd'hui, en insistant sur quelques points significatifs : le fait que les élites ne sont le plus souvent pas formées dans les universités, l'absence de décision qui caractérise ces dernières, la logique disciplinaire qui entrave - moins aujourd'hui - le processus d'autonomisation, la loi Edgar Faure de 1968 (en avance sur son temps), la Loi relative aux libertés et responsabilité des universités (LRU) et son impact, et les politiques actuelles d'excellence. Dans le second chapitre (pp. 27-45), Éric Piozin décrit la complexité de l'environnement institutionnel, la diversité et les disparités du réseau (voire du maquis) formé par les établissements, et les tentatives de rapprochement et de pilotage. L'auteur rappelle certains faits : deux tiers des étudiants seulement sont à l'Université, les publications sont en déclin. Parmi les faits plus positifs, le texte note la contribution de la mastérisation au rapprochement entre universités et (grandes) écoles, la constitution des pôles de recherche et d'enseignement supérieur (PRES), les fusions récentes (telles en Alsace et en Lorraine), la contractualisation (inaugurée dès 1984 
sous le ministère de l'Éducation nationale d'Alain Savary) qui couvre aujourdhui une large part des activités. Ce chapitre traite utilement des financements de I'Université, partis de très bas mais aujourd'hui en hausse. Le rattrapage prendra du temps : en France, les dépenses pour l'enseignement supérieur sont encore inférieures aux dépenses pour le secondaire, mais on a observé une prise de conscience générale de la nécessité de refinancer (et de refinancer autrement) I'Université. Dans le troisième chapitre (pp. 47-67), Jean-Pierre Finance traite de façon relativement claire de la gouvernance et de l'organisation des universités et s'attache à mettre en évidence certaines démarches cohérentes visant le bon fonctionnement du système, avec un regard sur l'avenir. Le texte comporte plus de langue de bois que les autres chapitres, de façon peutêtre inévitable. Parmi les changements relevés, notons que des universités plus autonomes et responsables peuvent aujourd'hui transformer des emplois d'enseignants en emplois techniques et vice versa. Le chapitre aborde en particulier les dysfonctionnements de la gouvernance, les batailles entre différentes hiérarchies, et les différentes logiques (disciplinaires, professionnelles, mixtes) ; il comporte une présentation succincte, mais bien venue, des laboratoires et écoles doctrinales, des services et livre, enfin, une analyse pertinente des changements, depuis la loi Edgar Faure jusqu'à la LRU, ses forces et ses faiblesses.

Dans la seconde partie, le quatrième chapitre de Daniel Filâtre (pp. 71-97) présente la formation des étudiants, leur répartition par territoires et disciplines, le rôle déterminant joué par le processus de Bologne et la généralisation des formations LMD (licence, maitrise, doctorat), les différences selon les types d'établissements, les réformes du diplôme d'études universitaires générales (DEUG) des années 90, les différentes étapes de la mise en œuvre de la réforme LMD et la formation continue. L'auteur analyse notamment les conséquences de la contractualisation et la réussite en licence. Dans le cinquième chapitre (pp. 99-126), Jean-Richard Cytermann aborde la recherche et les spécificités du système français dans ce domaine, notamment dues au fait qu'une grande partie de la recherche académique se fait en dehors de l'Université. Dans les années 80, la séparation de l'enseignement supérieur et de la recherche était encore généralisée, mais, aujourd'hui, $80 \%$ de l'activité des chercheurs au Centre national de la recherche scientifique (CNRS) et à l'Institut national de la santé et de la recherche médicale (INSERM) se fait dans les universités ainsi que dans les unités mixtes de recherche (UMR ). Cependant, on est loin de la prépondérance des universités comme au Royaume-Uni surtout et comme ailleurs en général. Le chapitre montre combien la recherche souffre d'un manque de visibilité et combien il est difficile de la mesurer, en raison notamment des doubles appartenances (par exemple à une université et à une grande école). II montre également comment on s'efforce aujourd'hui de mettre l'Université en position d'« arbitre entre enseignement et recherche » (p. I I2), au cœur de la recherche, et combien la mise en œuvre des évaluations par l'Agence d'évaluation de la recherche et de l'enseignement supérieur (AERES) a été décisive. Parmi les changements facilitateurs d'excellence, figurent aussi une redistribution plus cohérente des structures ministérielles, de nouvelles modalités de pilotage des établissements, une généralisation de la contractualisation, de nouvelles formes de collaboration avec les organismes extérieurs à l'Université et les initiatives d'excellence. Pour autant, la valorisation de la recherche reste notoirement insuffisante. Le chapitre suivant (pp. 127-143) permet à Guillaume Houzel de traiter de la vie étudiante, et de notamment relever la massification, les différenciations selon l'origine sociale et le sexe (les hommes prenant l'avantage sur les femmes au millieu des études supérieures). Le chercheur analyse le budget étudiant et la politique universitaire pour celui-ci (aide au logement, centres régionaux des œurres universitaires et scolaires - CROUS), caractérisée par une montée en puissance du rôle des collectivités locales. Le septième chapitre (pp. 145-159) porte sur linternational. Éric Froment revoit les conditions de mise en place des accords et les programmes tels Erasmus qui incitent à la mobilité des étudiants. Consulter le tableau présent en page 152 permet de constater que la France se situe au quatrième rang mondial pour les étudiants internationaux (un gros tiers de ces étudiants étant d'origine africaine), au même niveau environ que l'Allemagne, mais derrière le Royaume-Uni et loin derrière les Etats-Unis. Le texte signale que le contrôle qualité est loin d'être parfait, que la gouvernance est distante, et que linternational n'est pas au centre des préoccupations des universités. Le chapitre huit de Pierre Carbone (pp. |61-173) sur la politique documentaire détaille les relatives améliorations de celle-ci (les learning centres créant plus d'interactions parmi les étudiants en étant un exemple).

Dans la troisième partie, le neuvième chapitre de Claude Ronceray (pp. 177-189) traite de la gestion et des systèmes d'information, et mesure les changements importants qui résultent du transfert de la masse salariale de l'État aux établissements. II étudie le rôle structurant de la Loi organique relative aux lois de finances (LOLF) et de la révision générale des politiques publiques (RGPP), de l'importance d'un plan et d'un pilotage stratégiques prenant en compte les facteurs clés de succès dans chaque université, et de l'accent mis plus fréquemment 
sur le contrôle qualité. II résume également ce qui touche aux services informatiques et leurs réussites quand réussite il y a. Dans le dixième chapitre, Jean-Sébastien Valet et Gwenaëlle Verscheure (pp. 191-209) étudient les finances, et spécifiquement la façon dont les budgets sont préparés, les acteurs du financement, les nouvelles modalités du financement public, les conséquences de la LRU en matière de financement, la répartition des moyens à l'intérieur des universités, les nouveaux dialogues dans le domaine de la gestion et la professionnalisation des équipes budgétaires. Les auteurs relèvent que les avantages fiscaux (demi-part, par exemple) ne sont pas pris en compte dans le financement des universités, mais constituent une contribution non négligeable à leur financement, que l'hébergeur (souvent l'université) gère dorénavant l'ensemble des crédits de recherche et que la répartition des crédits se fait à un $1 / 5^{\mathrm{e}}$ en fonction de la performance - chiffre situé dans la fourchette haute de la moyenne européenne. On ne peut que déplorer le fait que le financement privé, la valorisation de la propriété intellectuelle, les contrats avec les entreprises, et le rôle des fondations soient si limités. Aussi la contribution plaide-t-elle pour une gestion pluriannuelle plus active. Le onzième chapitre de Frédéric Forest (pp. 21 I-224) traite des ressources humaines et des difficultés engendrées par la dispersion des établissements. II montre la répartition entre professeurs et autres personnels d'après des statistiques récentes, analyse brièvement les missions, les carrières et les rémunérations des enseignants-chercheurs et des autres catégories de personnels. Le chapitre douze de Nicolas Gaillard et Franck Joyeux (pp. 225-243) traite du patrimoine immobilier, souvent en mauvais état et difficile à gérer, des débuts de la dévolution de la propriété immobilière aux universités, de la rénovation prioritaire de certains pôles d'excellence et de campus innovants (moins de $10 \%$ des sites).

L'épilogue de (Frédéric Forest (pp. 245-259) présente l'Université comme un réseau reliant des points du système (acteurs, institutions) et animé par des flux (notamment financiers) dont la régulation a récemment évolué. II donne certaines orientations générales et transversales et insiste sur l'importance accrue de l'international, de la compétition, sur la question de l'égalité entre étudiants, sur les changements des flux financiers que régulent de plus en plus les universités et, enfin, sur la nécessité de continuer à changer le système.

Christophe Ippolito

Écritures, université de Lorraine, F-57000 christophe.ippolito@modlangs.gatech.edu

\section{Clément Hamel, Simon Malllard, Patrick Vassort, Le sport contre la société.}

Lormont, Éd. Le Bord de l'eau, coll. Altérité critique, $2012,200 \mathrm{p}$.

Alors que le sport occupe un espace de plus en plus important sur les écrans de télévision, d'ordinateurs, de tablettes et de smartphones, quill fait l'objet d'une médiatisation et d'une marchandisation sans précédent, la plupart des sociétés occidentales se ressentent encore des effets de la crise financière qui a durement frappé le monde entier et qui, visiblement, n'a pas eu le moindre impact sur le milieu du sport professionnel, pas plus d'un point de vue économique que politique. L'ouvrage est dirigé conjointement par Clément Hamel, Simon Maillard et Patrick Vassort, respectivement titulaire d'un master recherche en sciences et techniques des activités physiques et sportives et inscrit en master 2 de sociologie, doctorant en sociologie et maitre de conférences habilité à diriger des recherches en sociologie, tous trois au sein de l'université de Caen Basse-Normandie. L'étude va à l'encontre de l'idéologie dominante et de la ferveur populaire et médiatique autour du sport. Les trois directeurs de cette publication analysent le sport comme l'institution centrale d'une société capitaliste qui vend du rêve et des héros ou héroïnes inaccessibles (il n'y a qu'à voir les centaines de millions d'euros déboursés par des clubs de football européens pour acheter quelques joueurs soit connus, soit en devenir), alors même que le chômage se développe de manière exponentielle, que les populations s'appauvrissent et que les services publics d'éducation, de santé et de transport sont violemment critiqués. Pire encore, les auteurs perçoivent le sport comme le refuge idéologique de tous les courants politiques qui voient en lui fraternité, démocratie et mérite à tel point que, pour détourner le regard de l'opinion de la crise financière qui sévit, il devient impératif d'organiser des compétitions sportives internationales de grande ampleur fournissant un spectacle de qualité et des émotions fortes.

Dans l'introduction (pp. 9-12), les trois principaux auteurs écrivent : « L'institution sportive, depuis son avènement, bien que différemment selon les périodes, a toujours été l'appareil idéologique le plus puissant des idéologies dominantes, l'appareil de domination le plus fin et le plus acéré » (p. 9). Clément Hamel, Simon Maillard et Patrick Vassort apparentent le sport pratiqué d'une façon professionnelle à une forme de drogue à accoutumance, à un puissant instrument de coercition et de manipulation de la population que l'on abreuve de fables, de récits enchanteurs sur les valeurs du sport, le dépassement de soi et le sens de l'effort. Adoptant une posture résolument polémique, 